\title{
Study on Dynamic Guidance Obstacle Avoidance of UAV based on the Minimum Angle Shift
}

\author{
Xiong zhiyong ${ }^{1}$, Yang Xiuxia ${ }^{2}$, Zhang $\mathrm{Yi}^{2}, \mathrm{Hua} \mathrm{Wei}^{2}$, Liu Xiaowei ${ }^{2}$ \\ ${ }^{1}$ Science and Technology on Avionics Integration Laboratory, \\ Shanghai, 200233, China \\ ${ }^{2}$ Department of Control Engineering, Naval Aeronautical and Astronautical \\ University, Yantai, China \\ yangxiuxia@126.com
}

\begin{abstract}
With the extensive use of UAV, how to make the UAV avoid the obstacle and arrive at the destination has become a hot issue. To make the angle shift minimum, a method on dynamic guidance obstacle avoidance of UAV based on the minimum angle shift is proposed, considering the constraints such as the completion time of obstacle, the motor performance, the direction of velocity after obstacle avoidance and so on, determining the range of guidance parameters. In the meanwhile, the obstacle method is extended to $3 D$-space based on 2D. Moreover, numerical simulations are carried out to verify the effectiveness of the method.
\end{abstract}

Keywords: UAV; dynamic guidance obstacle avoidance; minimum angle shift

\section{Introduction}

The online flight planning of UAV (Unmanned Aerial Vehicle) must includes the avoidance of unknown threats. With the extensive use of UAV, how to make the UAV avoid the obstacle and arrive at the destination has become a hot issue.

To solve the problem of dynamic obstacle ovoidance, in paper [1,2], the concept of velocity obstacle is provided. The relative velocity information is solved and the position obstacle is transformed to velocity obstacle.

Paper[3] proposes the obstacle avoidance method based on the proportional navigation ratio. The method is easy to operate with good real-time performance, but the velocity direction of UAV cannot be predicted accurately due to the real-time variation of obstacle avoidance vector during the obstacle avoidance process, so the minimum angle shift cannot be guaranteed in most cases.

Hyo-Sang Shin[4,5] conducted research on real-time dynamic obstacle avoidance and collision avoidance method in the flight process based on differential geometry, applied velocity cone method to detect obstacle and avoid the obstacle based on the guidance law. But the non-continuous guidance law is given, and the symbolic function is introduced, so the planning path is prone to tremor.

Anusha Mujumdar[6] gave two nonlinear obstacle avoidance method based on the guidance law as well: nonlinear geometric guidance method and differential geometric guidance method, which can align the velocity vector of UAV at the target point. Moreover, the 3D-guidance law is given too.

F. Belkhouche[7-10] conducted 3D-navigation collision avoidance with extended proportional navigation ratio based on the velocity cone method, the constraints such as the flight velocity and angular speed is taken into consideration when design the guidance parameters, the method can give nonlinear guidance path. But the static obstacle is 
considered only, and the dynamic obstacle and the continuous flight path after obstacle avoidance is not studied.

To minimize the degree of the guidance path, the dynamic guidance obstacle avoidance method based on the minimum angle shift is given in the paper on the basis of the paper $[9,10]$. The paper $[9,10]$ solved the fixed point obstacle avoidance problem for static obstacle only, which lead the robot to a certain point beyond the obstacle to complete obstacle avoidance without considering the dynamic obstacle and the velocity vector of robot after obstacle avoidance. There are many question to apply the obstacle avoidance method to the collision avoidance problem of UAV directly, especially the dynamic obstacle avoidance, for the UAV has constraints such as high speed, minimum turning radius, no-jerk and so on. So the obstacle avoidance guidance method of UAV based on the minimum angle shift is given in the paper, which takes the completion time, the motor performance and the velocity vector after the obstacle avoidance into consideration and determines the range of guidance parameters.

\section{The Judgment of Collision and the Establishment of Kinematics Equations}

\subsection{Collision Judgment}

When obstacle is observed in the motor process, the geometric relationship of the UAV and the obstacle is given as Figure.1

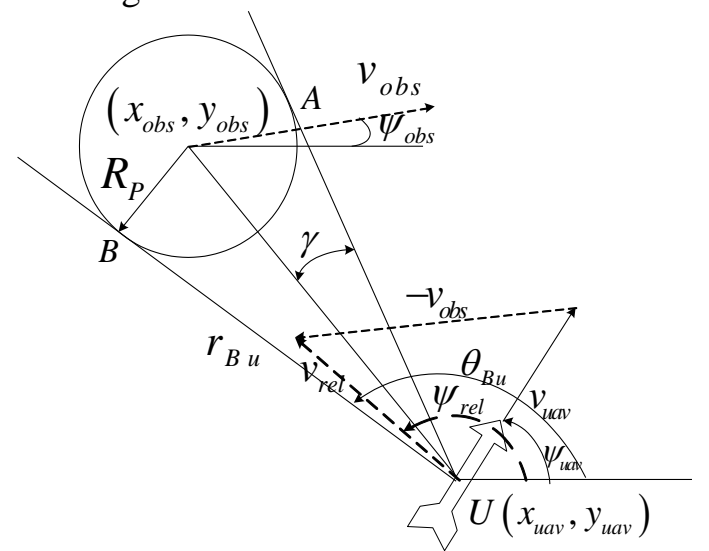

Figure 1. Geometric Relationship of the UAV and the Obstacle

Suppose the UAV with velocity $v_{\text {uav }}$ and path angle $\psi_{\text {uav }}$, the obstacle with velocity $v_{o b s}$ and path angle $\psi_{o b s}$. The relative velocity of UAV to obstacle is $v_{r e l}$, the relative path angle is $\psi_{\text {rel }}$. The UAV can be simplified as a particle $U$ by expanding the radius of obstacle circle, and then we establish collision zone $U A B$ based on the particle $U$ and the tangent line $U B 、 U A$.From the principle of speed obstacle we can know, if the relative velocity angle $\psi_{\text {rel }}$ meets the following condition:

$\theta_{B u}-2 \gamma<\psi_{r e l}<\theta_{B u}$

There will be collision risk between UAV and obstacle, so we need to adjust the velocity and path angle of UAV to make the relative speed $\vec{v}_{\text {rel }}$ outside the collision cone $U A B$ to complete the collision avoidance. When UAV found dynamic obstacles and need to adjust the speed to avoid obstacle, the UAV will adjust the speed to the tangential direction based on the minimum relative velocity angle shift, according to Figure 1, we select $U B$ as obstacle avoidance tangent line, that is fixed point $B$ is collision avoidance 
point.

\subsection{The Establishment of Kinematics Equations}

We can derive the kinematics equations between UAV and obstacle avoidance point $B$ base on the polar coordinates system shown as Figure.2.

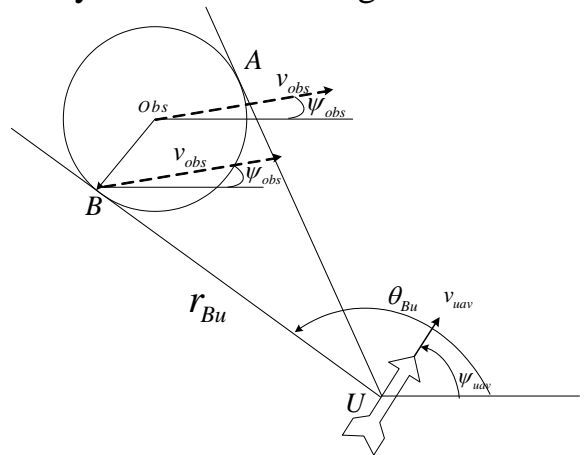

Figure 2. Kinematic Relationship of the UAV and the Obstacle

The expression of range change rate $\dot{r}_{B u}$ and angular velocity $\dot{\theta}_{B u}$ between UAV and obstacle avoidance point $B$ is as following:

$\dot{r}_{B u}=v_{o b s} \cos \left(\theta_{B u}-\psi_{o b s}\right)-v_{u a v} \cos \left(\theta_{B u}-\psi_{u a v}\right)$
$\dot{\theta}_{B u} r_{B u}=v_{u a v} \sin \left(\theta_{B u}-\psi_{u a v}\right)-v_{o b s} \sin \left(\theta_{B u}-\psi_{o b s}\right)$

For $\vec{v}_{\text {rel }}=\vec{v}_{\text {uav }}-\vec{v}_{\text {obs }}$, so the obstacle point $B$ can be considered stationary if we make the relative speed of UAV as $\vec{v}_{r e l}$ in the kinematics equations, and then the kinematics equations is as following:

$\dot{r}_{B u}=-v_{r e l} \cos \left(\theta_{B u}-\psi_{r e l}\right)$

$\dot{\theta}_{B u} r_{B u}=v_{r e l} \sin \left(\theta_{B u}-\psi_{\text {rel }}\right)$

\section{The Obstacle Avoidance Guidance Strategy based on the Minimum Angle Shift}

\subsection{The Design of Obstacle Avoidance Guidance Law based on the Minimum Angle Shift}

We design the obstacle avoidance guidance law based on the minimum angle shift on the basis of proportional navigation ratio $\dot{\psi}_{r e l}=N \dot{\theta}_{B u}$, in where $N$ is proportional navigation coefficient and $N>1$.

For we suppose the relative velocity of UAV is $\vec{v}_{r e l}$ and the obstacle is stationary, when $N>2\left|\dot{r}_{B u}\right| / v_{r e l} \cos \left(\theta_{B u}-\psi_{r e l}\right)=2,\left|\dot{\theta}_{B u}\right|$ will decrease gradually and tend to zero, so the proportional navigation coefficient need meet $N>2$ to guarantee angle stability at the obstacle point $B$.

When the proportional navigation coefficient is confirmed, we consider the initial status:

(1)If $\psi_{\text {rel }}\left(t_{0}\right)=N \theta_{B u}\left(t_{0}\right)$, the relative velocity angle $\psi_{\text {rel }}(t)$ guidance law is shown as following:

$\psi_{\text {rel }}(t)=N \theta_{B u}(t)$ 
(2) If $\psi_{\text {rel }}\left(t_{0}\right) \neq N \theta_{B u}\left(t_{0}\right)$, the relative velocity angle $\psi_{\text {rel }}(t)$ guidance law is shown as following:

$$
\psi_{\text {rel }}(t)=N \theta_{B u}(t)+\psi_{\text {rel }}\left(t_{0}\right)-N \theta_{B u}\left(t_{0}\right)
$$

Suppose $c_{0}=\psi_{\text {rel }}\left(t_{0}\right)-N \theta_{B u}\left(t_{0}\right)$, the formula (7) can be expressed as following:

$$
\psi_{\text {rel }}(t)=N \theta_{B u}(t)+c_{0}
$$

$c_{0}$ is constant deviation angle, which control the final path angle of UAV under the action of guidance law, the stability of guidance law will be proved in chapter3.2.

The formula (4), (5) can be expressed as following based on the guidance equation:

$$
\begin{aligned}
& \dot{r}_{B u}=-v_{r e l} \cos \left[(1-N) \theta_{B u}-c_{0}\right] \\
& \dot{\theta}_{B u} r_{B u}=v_{r e l} \sin \left[(1-N) \theta_{B u}-c_{0}\right]
\end{aligned}
$$

But under most cases, when the proportional navigation coefficient $N$ and the constant deviation angle $c$ are confirmed, the initial path angle $\psi_{\text {rel }}\left(t_{0}\right)$ of UAV cannot meet the equation $\psi_{\text {rel }}\left(t_{0}\right)=N \theta_{B u}\left(t_{0}\right)+c$, so the designed guidance law cannot be applied directly.

To solve the problem, the time-varying deviation angle function is added to the liner guidance law to conduct pre-conditioning so as to satisfy the given guidance law. In the meanwhile, the adjusting process is limited in a finite time, that is the time-varying deviation angle function need converge to a minimum $\varepsilon \leq 10^{-4}$ in a finite time to confirm the proportional navigation coefficient $N$ and the constant deviation angle $c$.

The exponential function is selected as time-varying deviation angle function to conduct pre-conditioning, time-varying deviation angle function is shown as following:

$$
f(t)=b_{0} e^{-b_{1} t}
$$

In where, $b_{1}$ is positive to guarantee the attenuation of exponential function.

For $t=0, f(0)=b_{0}$, so the coefficient $b_{0}$ is the deviation between the initial path angle $\psi_{\text {rel }}\left(t_{0}\right)$ and $N \theta_{B u}\left(t_{0}\right)+c$, that is

$$
b_{0}=\psi_{r e l}\left(t_{0}\right)-N \theta_{B u}\left(t_{0}\right)-c
$$

The minimum angle shift guidance law with time-varying deviation angle function is shown as following:

$$
\psi_{\text {rel }}(t)=N \theta_{B u}(t)+c+b_{0} e^{-b_{1} t}
$$

Now we confirm the range of coefficient $b_{1}$.

From the adjustment function requirements, when UAV arrives at obstacle point $B$, the index regulation process is over.

Suppose the time of UAV to reach obstacle point $B$ is $T_{\text {reach }}$, when $t=T_{\text {reach }}$ in formula (16)

$f\left(T_{\text {reach }}\right)=b_{0} e^{-b_{1} T_{\text {reach }}}<\varepsilon$

Where, $\varepsilon$ is minimum value, which meets $\varepsilon \leq 10^{-4}$.

For $b_{0}$ is known, so the lower limit value of $b_{1}$ according to formula(14) is

$b_{1}>\ln \left(b_{0} / \varepsilon\right) / T_{\text {reach }}$

And we can get from the formula(11) derivation 
$f^{\prime}(t)=b_{0}\left(-b_{1}\right) e^{-b_{1} t}$

So we can know that $f^{\prime}(t)$ will converge to a minimum $\varepsilon \leq 10^{-4}$ in a finite time, and $f^{\prime}(t)$ is a part of UAV's angular velocity, when $t=0$

$f^{\prime}(0)=b_{0}\left(-b_{1}\right)$

Suppose the relative velocity $v_{r e l}$ of UAV is invariant, so the normal acceleration needed in the guidance process is $a=v_{r e l} \dot{\psi}_{\text {rel }}$, and the upper limit value of $b_{1}$ can be got under overload constraint.

Suppose the permissible load factor of UAV is $n$, we can get

$v_{\text {rel }} \dot{\psi}_{\text {rel }} \leq n \times g$

Where, $g$ is the acceleration of gravity.

For $\left|f^{\prime}(0)\right|<\left|\dot{\psi}_{\text {rel }}(0)\right|$, so

$b_{1}<\frac{n \times g}{b_{0} \times v_{r e l}}$

Combined with formula (15),(19), we can get the range of coefficient $b_{1}$ is

$\ln \left(b_{0} / \varepsilon\right) / T_{\text {reach }}<b_{1}<\frac{n \times g}{b_{0} \times v_{\text {rel }}}$

Suppose $r$ is turning radius in the adjustment process, so

$$
\dot{\psi}_{r e l} \times r=v_{r e l}
$$

We can know the larger $b_{1}$, the smaller $r$. So the size of turning radius $r$ of UAV depends on $b_{1}$, and turning radius is time varying.

Now we can get the range of guidance coefficient $N$ and $b_{1}$ meeting the overload constraints and stability of relative velocity angle in the final stage, and we can design reasonable guidance law.

In the design of guidance law, the purpose of exponential function is to adjust the path angle $\psi_{\text {rel }}$ with its attenuation. So there two process under the guidance law.

(1) The flight process based on the time-varying deviation angle function is pre-adjustment process, we can know the radius of curvature time varying from formula (16).

(2) The flight process based on the constant deviation angle, the deviation angle is constant when adjustment function is minimum. With guidance law, the relative velocity angle will converge to a required constant value when UAV arrived at the destination. The proof is in chapter3.2.

\subsection{Stability Proof}

The equation (4),(5)is shown as following based on the guidance law(13):

$$
\begin{aligned}
& \dot{r}_{B u}=-v_{r e l} \cos \left[(1-N) \theta_{B u}-c-b_{0} e^{-b_{1} t}\right] \\
& \dot{\theta}_{B u}=\frac{v_{r e l} \sin \left[(1-N) \theta_{B u}-c-b_{0} e^{-b_{1} t}\right]}{r_{B u}}
\end{aligned}
$$

We know that the exponential term $b_{0} e^{-b_{1} t} \rightarrow 0$ with time $t \rightarrow \infty$ for $b_{1}>0$.

So we can get the balanced point of $\theta_{B u}$ when $\dot{\theta}_{B u}=0$ based on the formula(22). 
$\theta_{B u}^{e q 1}=\frac{c}{1-N}$ or $\theta_{B u}^{e q 2}=\frac{c}{1-N}+\pi$

We can get based on the liner theory,

$\left.\frac{\partial \dot{\theta}_{B u}}{\partial \theta_{B u}}\right|_{\theta_{B u}^{e q 1}}=\frac{v_{r e l}}{r_{B u}}(1-N)=A$

$\left.\frac{\partial \dot{\theta}_{B u}}{\partial \theta_{B u}}\right|_{\theta_{B u}^{e q 2}}=-\frac{v_{r e l}}{r_{B u}}(1-N)=B$

Where $A<0, B>0$ for $v_{r e l}>0, r_{B u}>0, N>1$, so we can know $\theta_{B u}^{e q 1}$ is global stability point, which is $t \rightarrow \infty, \theta_{B u} \rightarrow \theta_{B u}^{e q 1}$.

$\dot{r}_{B u} \rightarrow-v_{\text {rel }}$ when $\theta_{B u} \rightarrow \theta_{B u}^{e q 1}$, which reflects that the distance reduce gradually and the $\mathrm{UAV}$ arrives at the destination finally.

Therefore, when UAV approach the obstacle point $B$, the relative velocity angle converges to $\theta_{B u}^{e q 1}$. The obstacle avoidance is complete if we select appropriate guidance parameter to make the relative path angle converges to $\theta_{B u}^{e q 1}$.

So the design of obstacle avoidance algorithm is as following:

(1) If the relative velocity $v_{\text {rel }}$ of UAV to obstacle is in the collision cone when UAV finds obstacle, the UAV need take measure to avoid obstacle. It is better with smaller path shift considering the optimal path of UAV based on an optimal index.

(2) To work the tangent angle of the obstacle circles to obstacle point $B$.

(3) Design the guidance law equation(13), determine the appropriate parameters $N$ 、 $c 、 b_{0} 、 b_{1}$ to meet the initial constraints.

$\psi_{\text {rel }}\left(t_{0}\right)=N \theta_{B u}\left(t_{0}\right)+c+b_{0}$

And to make the relative path angle $\psi_{\text {rel }}\left(t_{f}\right)$ converge to $\theta_{B u}^{e q 1}$ and equal to the initial tangent direction angle $\theta_{B u}\left(t_{0}\right)$ of $U B$, that is $\psi_{\text {rel }}\left(t_{f}\right)=\theta_{B u}^{e q 1}=\theta_{B u}\left(t_{0}\right)$, under the guidance law.

In this way, the UAV can arrive at the obstacle point successfully and the angle meets the collision avoidance requirements.

\subsection{Velocity Solution of UAV}

We design the guidance law on the relative velocity angle $\psi_{\text {rel }}(t)$ to make the UAV avoid the obstacle. Suppose the relative velocity $v_{\text {rel }}$ is invariant, so the acceleration of relative velocity is:

$a_{\text {rel }}=v_{\text {rel }} \times \dot{\psi}_{\text {rel }}$

And suppose the obstacle is static or in a uniform motion, so the acceleration of relative velocity $a_{r e l}$ is offered by UAV wholly, the schematic of relationship between the $v_{r e l}$ with the $v_{u a v}$ and the $v_{o b s}$ is shown as following: 


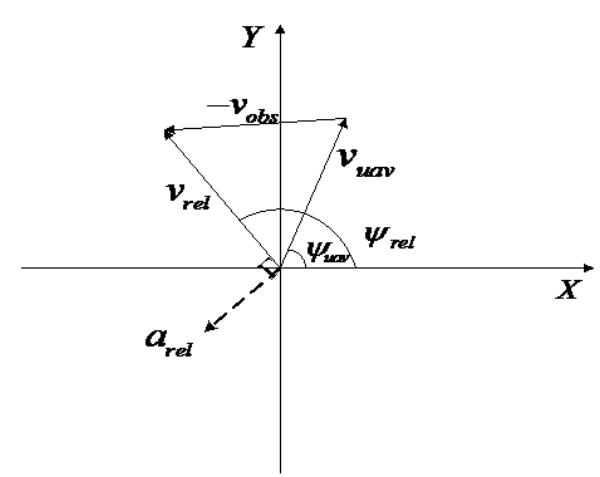

Figure 3. Schematic of Relationship between the $v_{r e l}$ with the $v_{u a v}$ and the $v_{o b s}$

So we can get the variance ratio of velocity and path angle:

$\dot{v}_{\text {uav }}(t)=-a_{\text {rel }}(t) \sin \left(\psi_{\text {rel }}(t)-\psi_{\text {uav }}(t)\right)$

$\dot{\psi}_{\text {uav }}(t)=a(t) \cos \left(\psi_{\text {rel }}(t)-\psi_{\text {uav }}(t)\right) / v_{\text {uav }}(t)$

And we can get the moving track of UAV from equation (27) and (28).

\subsection{Simulation Analysis}

Suppose UAV finds obstacle in 2D-plane motion, the initial condition of UAV and obstacle is shown in table 1 . The radius of UAV and obstacle is supposed as $R_{P}=2 \mathrm{~km}$ and the obstacle is supposed in uniform motion, and the permissible load factor is $n=5$.

Table 1. Initial Condition of the UAV and the Obstacle

\begin{tabular}{|c|c|c|c|}
\hline & $(x, y)$ & $v$ & $\psi$ \\
\hline UAV & $(0 \mathrm{~km}, 0 \mathrm{~km})$ & $200 \mathrm{~m} / \mathrm{s}$ & $\pi / 2 \mathrm{rad}$ \\
\hline Obstacle & $(-10 \mathrm{~km}, 10 \mathrm{~km})$ & $200 \mathrm{~m} / \mathrm{s}$ & $0 \mathrm{rad}$ \\
\hline
\end{tabular}

Combine the initial condition with Figure 1, we can get the initial distance between UAV and obstacle is $R_{0}=10 \sqrt{2} \mathrm{~km}$, the relative velocity is $v_{r e l}=200 \sqrt{2} \mathrm{~m} / \mathrm{s}$ and is invariant during the fight process. The initial distance between UAV and obstacle point $B$ is $r_{B u 0}=13.6 \mathrm{~km}$, the initial sight angle is $\theta_{B u}\left(t_{0}\right)=2.643 / \mathrm{rad}$, the time to reach the obstacle is $T_{\text {reach }} \approx r_{\text {Bu } 0} / v_{\text {rel }}=48 \mathrm{~s}$.

And then we select the guidance parameter as: $N=4, b_{1}=0.15$, $c=-(N-1) \theta_{B u}\left(t_{0}\right), b_{0}=\psi_{\text {rel }}-\theta_{B u}\left(t_{0}\right)$ from the constraints of collision avoidance completion time, coefficient of proportionality $N>2$, the maximum normal overload of $\mathrm{UAV}$ and the global stability point of sight angle $\theta_{B u}^{e q 1}$.

The simulation result is shown as Figure.4 to Figure. 9. 


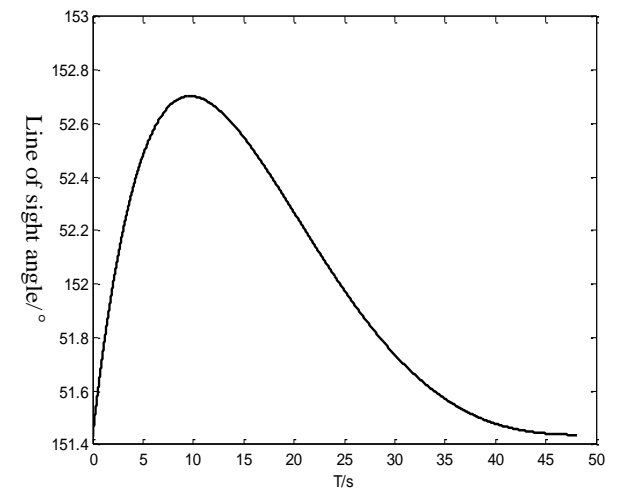

Figure 4. Schematic of Sight Angle

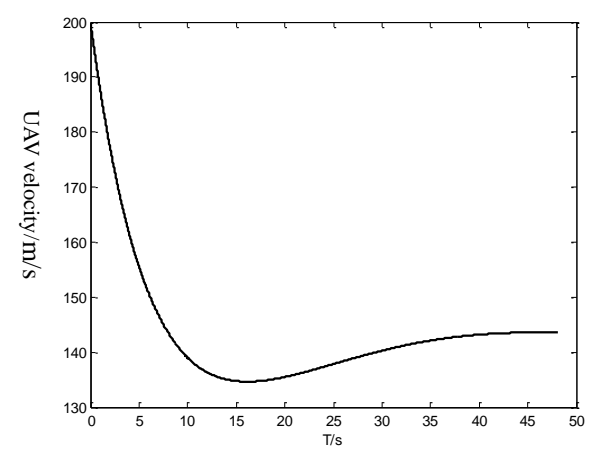

Figure 6. The Magnitude of the UAV Velocity

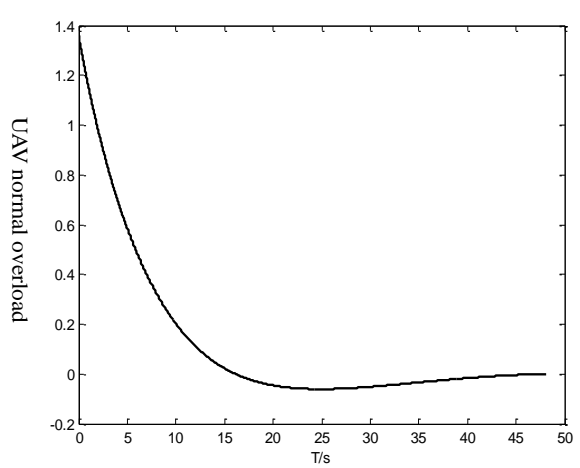

Figure 8. The Required Normal Overload of UAV

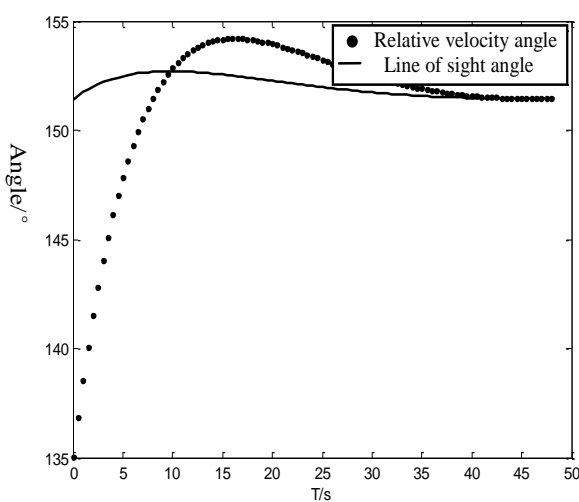

Figure 5. Schematic of Relative Velocity Angle

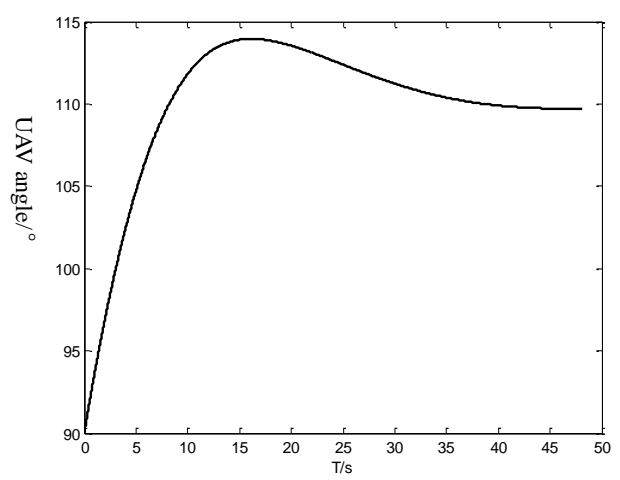

Figure 7. The Heading Angle of the UAV Velocity

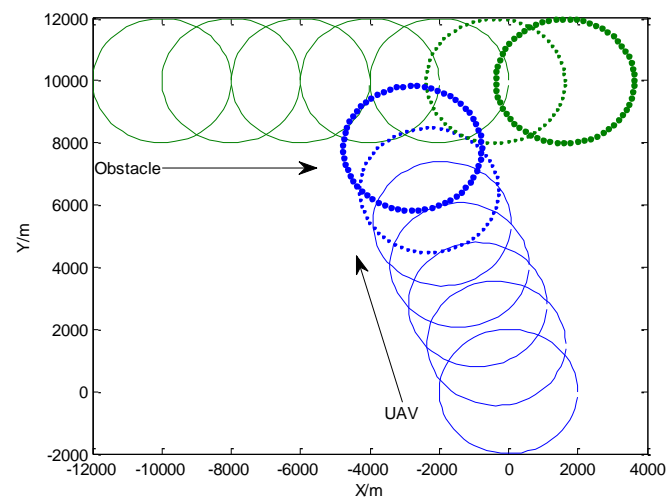

Figure 9. The Movement of the UAV Avoiding the Obstacle

From the Figure 4 the schematic of sight angle we can get the sight angle is equal to initial sight angle $\theta_{B u}\left(t_{0}\right)$ when $t=48 \mathrm{~s}$, and from Figure.5, we know the relative velocity angle $\psi_{r e l}$ is in coincidence with sight angle $\theta_{B u}\left(t_{0}\right)$ and equal to each other, which reflects that the relative velocity angle has moved to tangent direction and UAV can reach the obstacle point $B$ and the correctness of obstacle avoidance algorithm, from Figure.6, Figure.7 and Figure.8, we can get the maximum normal overload of UAV is 
$n=1.4$, which is less than maximum normal overload to ensure the change of velocity and direction, from Figure.9, we can know the initial path angle $\psi_{\text {uav } 0}=\pi / 2$ is counterclockwise rotation under the minimum angle shift guidance law, and contact with obstacle(shown as the first dotted circle) with larger distance (shown as the second dotted circle), which reflects the success of collision avoidance.

\section{Study on 3D Obstacle Avoidance}

\subsection{Study on Dimensionality Reduction Obstacle Avoidance}

3D obstacle avoidance algorithm of UAV based on the minimum angle shift guidance law is designed, the paper[10] extend the obstacle avoidance algorithm from 2D plane to 3D plane(shown as Figure.10)to solve the 3D obstacle avoidance problem.
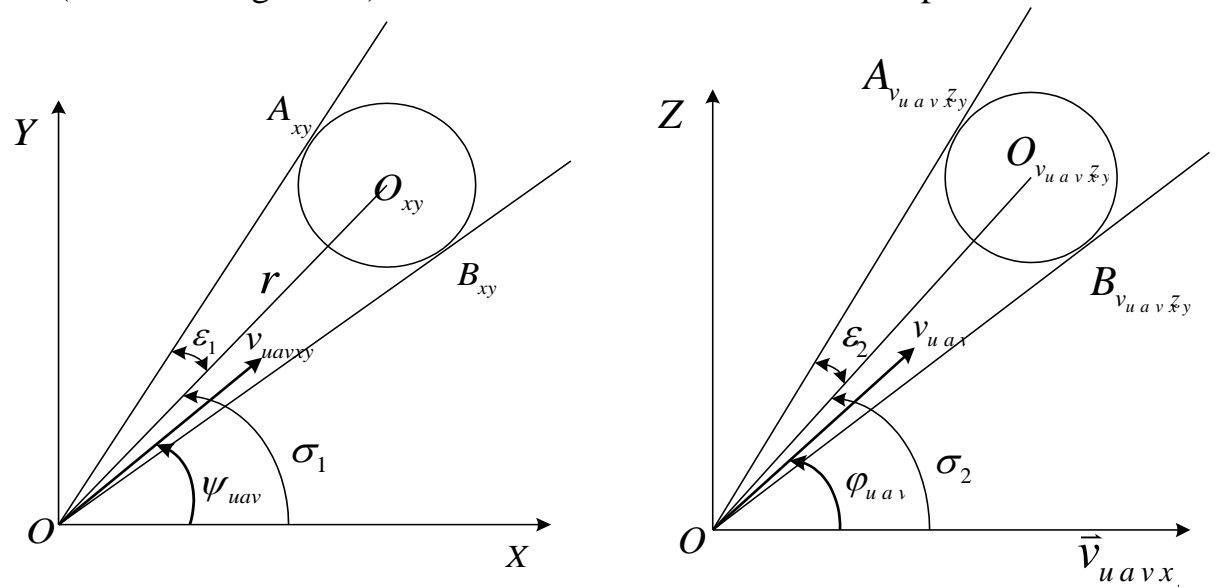

Figure 10. The Collision Threat Schematic between UAV and Obstacle in 3D Plane

We can design the path angle $\psi_{\text {uav }}$ or track angle $\varphi_{\text {uav }}$ based on the guidance law to make UAV reach the obstacle point and meet the obstacle avoidance requirements when the obstacle avoidance point is confirmed.

\subsection{The Simulation Analysis}

Suppose UAV finds obstacle in 3D-plane motion, the initial condition of UAV and obstacle is shown in table 2. The radius of UAV and obstacle is supposed as $R_{P}=2 \mathrm{~km}$ and the obstacle is supposed in uniform motion, and the permissible load factor is $n=5$.

Table 2. The Initial Conditions of the UAV and the obstacle

\begin{tabular}{|c|c|c|c|c|}
\hline & $(x, y, z)$ & $v$ & $\psi$ & $\varphi$ \\
\hline UAV & $(0 \mathrm{~km}, 0 \mathrm{~km}, 0 \mathrm{~km})$ & $100 \mathrm{~m} / \mathrm{s}$ & $\pi / 4 \mathrm{rad}$ & $\arctan (\sqrt{2} / 2) \mathrm{rad}$ \\
\hline Obstacle & $(10 \mathrm{~km}, 10 \mathrm{~km}, 10 \mathrm{~km})$ & $100 \mathrm{~m} / \mathrm{s}$ & $5 \pi / 4 \mathrm{rad}$ & $(\arctan (\sqrt{2} / 2)+\pi) \mathrm{rad}$ \\
\hline
\end{tabular}

From the relationship of UAV and obstacle, we can get the initial velocity of UAV to obstacle is $v_{\text {rel0 }}=200 \mathrm{~m} / \mathrm{s}, \psi_{\text {rel0 }}=\pi / 4, \varphi_{\text {rel0 }}=\arctan (\sqrt{2} / 2) / \mathrm{rad}$, and there is 
collision threat between UAV and obstacle.

Suppose the UAV carried the obstacle avoidance by path angle shift.

First select the obstacle avoidance point. Combined with Figure.10, we select $B_{x y}$ as obstacle avoidance point. And we can get the distance between UAV and obstacle is $R_{0}=10 \sqrt{2} \mathrm{~km}$, the distance between $B_{x y}$ and UAV is $r_{0}=13.6 \mathrm{~km}$, the initial sight angle of UAV to obstacle is $\sigma_{1}=\left(\pi / 4-\arcsin \left(2 \times R_{P} / R_{0}\right)\right) / \mathrm{rad}$, the time of UAV to reach horizontal obstacle point $B_{x y}$ is $T_{\text {reach }}=r_{0} /\left(v_{\text {rel } 0} \cos \varphi_{\text {rel0 }}\right)=85 \mathrm{~s}$

The guidance law parameter is designed as following under the constraints of time, motion performance and path angle after obstacle avoidance.

$$
\begin{array}{ll}
N_{1}=4 \quad m_{1}=\arcsin \left(2 \times R_{P} / R_{0}\right) & \quad \\
c_{1}=-3 \times\left(\pi / 4-\arcsin \left(2 \times R_{P} / R_{0}\right)\right) 。 & d_{1}=0.09
\end{array}
$$

The analysis result is shown as Figure.11 to Figure.16.

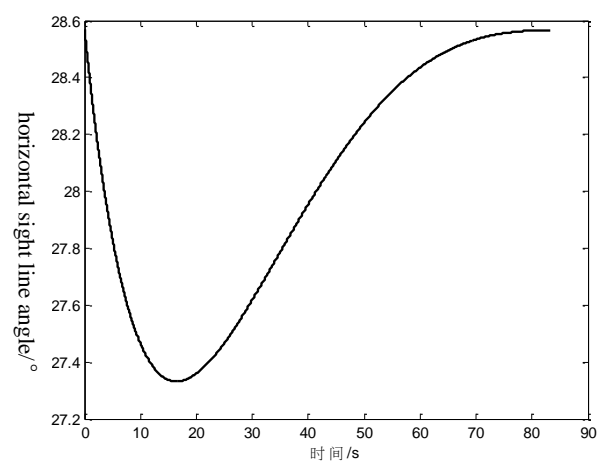

Figure 11. Angle of the Horizontal Sight Line

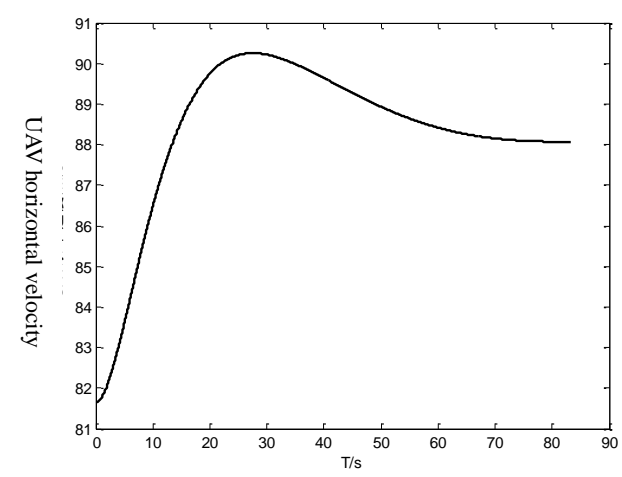

Figure 13. Magnitude of the Horizontal Velocity

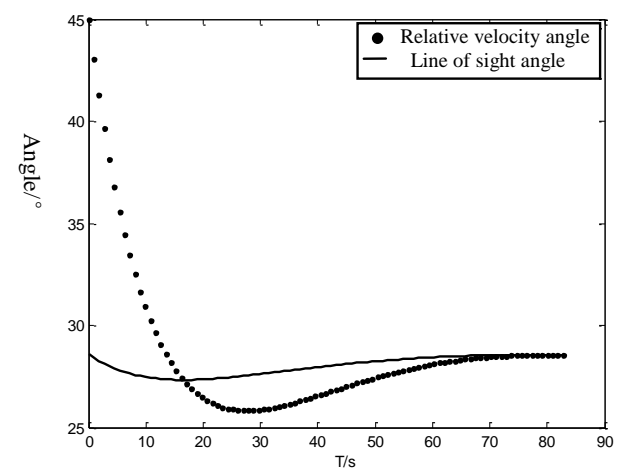

Figure 12. Heading Angle of the Relative Velocity

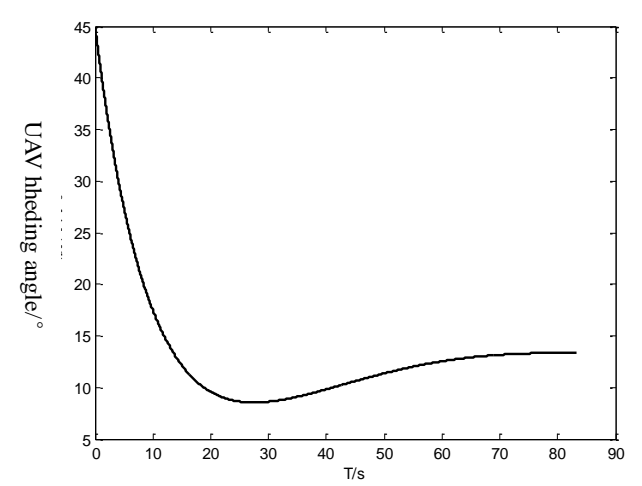

Figure 14. Heading Angle of the Velocity 


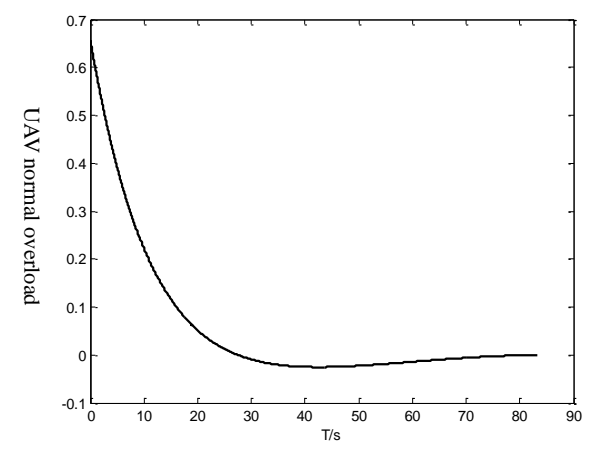

Figure 15. Required Normal

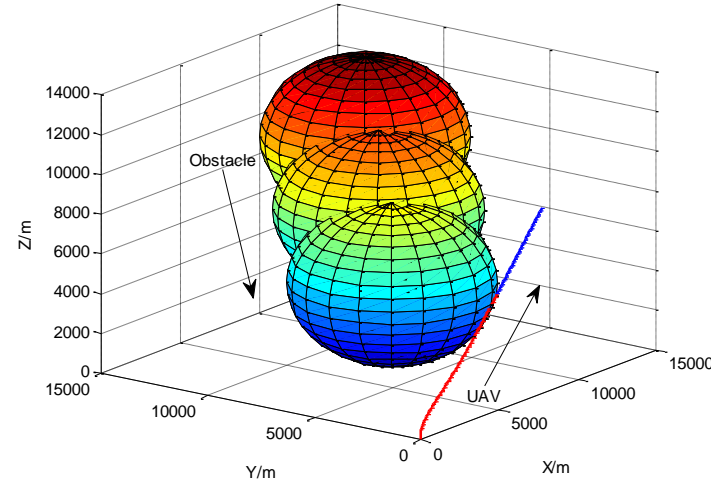

Figure 16. Movement of the UAV and the Obstacle

From the Figure.11 and Figure.12, the schematic of horizontal sight angle and the heading angle of the relative velocity, we can get the horizontal sight angle and the heading angle of the relative velocity converge to the direction of initial sight angle and complete the obstacle avoidance task. From Figure.13, Figure.14 and Figure.15, we can get the maximum normal overload of UAV is $n=0.65$, from Figure.16, we can know UAV contact with obstacle is we suppose the obstacle is static, so the obstacle avoidance algorithm is correct.

\section{Conclusion}

The dynamic guidance obstacle avoidance of UAV based on the minimum angle shift proposed in the paper includes three parts: the proportional term based on the sight angle, the constant deviation term and time-varying and exponential term. Considering the motor performance of UAV and completion time constraints, the range of the minimum angle shift guidance law parameters is work out adapted to UAV and is applied to the online obstacle avoidance algorithm design, which solved the velocity direction determination after obstacle avoidance and minimum angle shift problem to guarantee the path optimization. And in the same time, the problem is extended to 3D-plane from 2D-plane, the analysis result proves the effectiveness of obstacle avoidance algorithm. The obstacle avoidance algorithm is fit with the online obstacle avoidance in conflict environment for its simple principle and path optimization. And the next work is to study the online obstacle avoidance in multi-obstacle environment and obstacle avoidance between multi-UAV.

\section{Acknowledgements}

This research is supported by Aeronautical Science Foundation of China under Grant No20135584010.

\section{References}

[1] P. Fiorini, Z. Shiller, "Motion planning in dynamic environments using the relative velocity paradigm", IEEE International Conference on Robotics and Automation. Piscataway, NJ, USA: IEEE, (1993), pp. 560-565.

[2] P. Fiorini , Z. Shiller, "Motion planning in dynamic environments using velocity obstacles",International Journal of Robotics Research, vol. 17, no. 7, (1998), pp. 760-772.

[3] S C Han, H Bang, "Proportional navigation-based optimal collision avoidance for UAVs", 2nd international conference on autonomous robots and agents, Dec. (2004), pp. 356-369. 
[4] H-S Shin, A Tsourdos and B White, "UAS Conflict Detection and Resolution Using Differential Geometry Concepts". Sense and Avoid in UAS: Research and Applications, First Edition. Edited by Plamen Angelov. (2012) John Wiley \& Sons, Ltd. Published 2012 by John Wiley \& Sons, Ltd.

[5] B. A., White, H. S., Shin, and A Tsourdos, "UAV Obstacle Avoidance Using Differential Geometry Concepts". IFAC World Congress 2011, Milan, Italy, (2011), pp. 6325 6330.

[6] A Mujumdar, R Padhi, "Reactive Collision Avoidance Using Nonlinear Geometric and Differential Geometric Guidance", Journal of Guidance, Control, and Dynamics. vol. 34, no. 1, January-February (2011), pp. 303 310.

[7] F Belkhouche, "Nonholonomic robots navigation using linear navigation functions". Proceedings of the 2007 American Control Conference.July (2007), pp. 388 390.

[8] F.Belkhouche, B.Belkhouche, P.Rastgoufard, "Autonomous navigation and obstacle avoidance using navigation laws with time-varying deviation functions".Advanced Robotics. vol. 21, no. 5-6, (2007), pp. 555-581

[9] F Belkhouche, "Reactive path planning in a dynamic environment", IEEE Transactions on Robotics. vol. 25, no. 4, (2009), pp. 902-911.

[10] F. Belkhouche and B. Bendjilali. Reactive Path Planning for 3-D Autonomous Vehicles. IEEE Transactions on Control Systems Technology. Vol. 20, No. 1, JANUARY (2012), pp. 249-256.

\section{Authors}

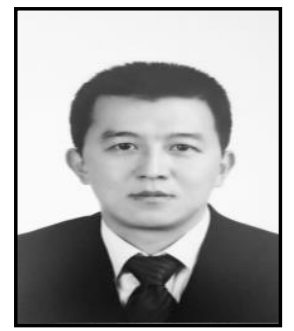

Xiong Zhiyong, was born in China in 1972. He received his master degree in Northwestern Polytechnical University, Xi'an, and China in 1997. Since 1997, he has been Science and Technology on Avionics Integration Laboratory, Shanghai, where he is currently a research professor. His main research interest is integrated avionics system.

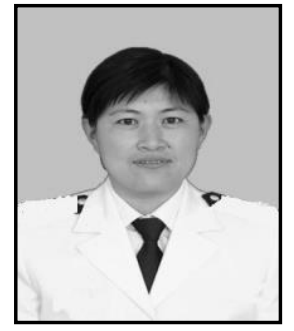

Yang Xiuxia, was born in Laizhou, Shandong Province, China in 1975. He received his Ph.D. degree in electrical engineering from naval university of engineering, Wuhan, China in 2005. Since 2000, he has been with department of control engineering of naval aeronautical and astronautical university, where he is currently a vice professor. His main research interests include nonlinear control theory with applications to robots, aircraft and other mechanical systems.

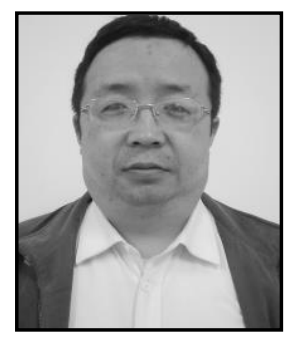

Zhang Yi, was born in Rongcheng, Shandong Province, China in 1971. He received his master degree in control theory and application from naval aeronautical and astronautical university, Yantai, China in 2001. Since 2000, he has been with department of control engineering of naval aeronautical and astronautical university, where he is currently a vice professor. His main research interests include nonlinear control theory with applications to robots, aircraft and other mechanical systems. 